\title{
Analysis of the protein-protein interaction networks of differentially expressed genes in pulmonary embolism
}

\author{
HAO WANG $^{1 *}$, CHEN WANG $^{1 *}$, LEI ZHANG $^{1}$, YINGHUA LU $^{1}$, QIANGLIN DUAN ${ }^{2}$, \\ ZHU GONG ${ }^{2}$, AIBIN LIANG ${ }^{3}$, HAOMING SONG ${ }^{2}$ and LEMIN WANG ${ }^{2}$ \\ Departments of ${ }^{1}$ Family Medicine, ${ }^{2}$ Cardiology and ${ }^{3}$ Hematology, Tongji Hospital, \\ School of Medicine, Tongji University, Shanghai 200065, P.R. China
}

Received January 6, 2014; Accepted October 24, 2014

DOI: $10.3892 / \mathrm{mmr} .2014 .3006$

\begin{abstract}
The aim of the present study was to explore the function and interaction of differentially expressed genes (DEGs) in pulmonary embolism (PE). The gene expression profile GSE13535, was downloaded from the Gene Expression Omnibus database. The DEGs 2 and $18 \mathrm{~h}$ post-PE initiation were identified using the affy package in $\mathrm{R}$ software. The Kyoto Encyclopedia of Genes and Genomes (KEGG) pathways of the DEGs were analyzed using Database for Annotation Visualization and Integrated Discovery (DAVID) online analytical tools. In addition, protein-protein interaction (PPI) networks of the DEGs were constructed using the Search Tool for the Retrieval of Interacting Genes/Proteins. The PPI network at $18 \mathrm{~h}$ was modularized using ClusterONE, and a functional enrichment analysis of the DEGs in the top three modules was performed with DAVID. Overall, 80 and 346 DEGs were identified 2 and $18 \mathrm{~h}$ after PE initiation, respectively. The KEGG pathways, including chemokine signaling and toll-like receptor signaling, were shown to be significantly enriched. The five highest degree nodes in the PPI networks at 2 or $18 \mathrm{~h}$ were screened. The module analysis of the PPI network at $18 \mathrm{~h}$ revealed 11 hub nodes. A Gene Ontology terms analysis demonstrated that the DEGs in the top three modules were associated with the inflammatory, defense and immune responses. The results of the present study suggest that the
\end{abstract}

Correspondence to: Dr Hao Wang, Department of Family Medicine, Tongji Hospital, School of Medicine, Tongji University, 389 Xincun Road, Shanghai 200065, P.R. China

E-mail: hao_wang@tongji.edu.cn

Dr Lemin Wang, Department of Cardiology, Tongji Hospital, School of Medicine, Tongji University, 389 Xincun Road, Shanghai 200065, P.R. China

E-mail: wanglemin@tongji.edu.cn

*Contributed equally

Key words: pulmonary embolism, protein-protein interaction network, functional modules, differentially expressed genes, pathways
DEGs identified, including chemokine-related genes TFPI2 and TNF, may be potential target genes for the treatment of $\mathrm{PE}$. The chemokine signaling pathway, inflammatory response and immune response were explored, and it may be suggested that these pathways have important roles in PE.

\section{Introduction}

After cancer and myocardial infarction, pulmonary embolism (PE) is the third-most-common cause of mortality in the United States (1,2), with $>600,000$ cases arising annually (3). The one-year mortality rate is $2.5 \%$ for treated PE (4) and $30 \%$ for untreated PE (5), according to previous research. Numerous studies have focused on the causes of PE. PE has been identified as the most serious complication of deep vein thrombosis (DVT) (6), and is defined as an embolus obstructing a vessel, or the outline of an embolus within a vessel $(7,8)$. DVT is closely associated with PE (9), as well as cardiac disease (10) and other chronic diseases (4). The presenting symptoms of PE are non-specific and final diagnosis is based on an angiogram (4).

Previous studies have identified genetic mutations that are associated with PE, such as Factor V Leiden (11), prothrombin factor II G20210A (12) and val34leu mutation in factor XIII (13). In addition, common polymorphisms of methylenetetrahydrofolate reductase $(14,15)$ appear to be associated with PE. Genes, such as plasminogen activator inhibitor-1 (PAI-1) that reduces fibrinolytic capacity (16) and granulocyte-macrophage colony stimulating factor (GM-CSF) that participates in the immune function by affecting the balance of $\mathrm{Th}$ (helper $\mathrm{T}$ cell) 1/Th2 (14,17), are reported as risk factors for PE. Furthermore, deficiencies of certain proteins, such as hereditary protein S (16), protein C and antithrombin III (12), have been reported to impact arterial thrombosis and PE. Although previous studies have identified numerous potential genes and proteins that may be the determinants of PE, it remains essential to research the potential etiology and pathogenesis of PE, due to its high incidence, high rate of mortality, high level of misdiagnosis and low detection rate.

The present study used microarrays to identify differentially expressed genes (DEGs) between specimens acquired 2 and $18 \mathrm{~h}$ following injection of a microsphere, and specimens acquired following injection of a control vehicle. Bioinformatics methods were used to construct protein-protein 
$\mathbf{A}$

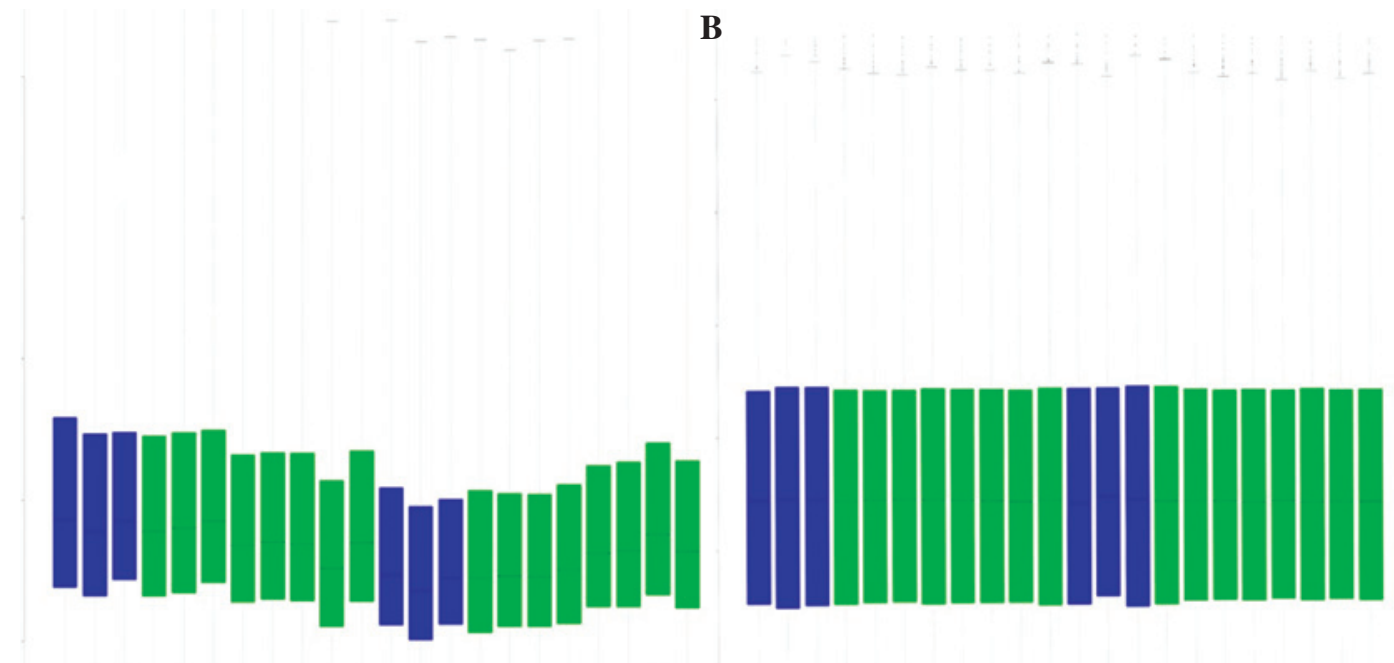

Figure 1. Box plots of the expression spectrum data (A) before and (B) after standardization. Blue represents the control group and green represents the experimental groups.

interaction (PPI) networks, and the functional modules in the networks were analyzed.

\section{Materials and methods}

Derivation of genetic data. The gene expression profile of GSE13535 (18) was downloaded from the Gene Expression Omnibus (GEO) database (http://www.ncbi.nlm.nih.gov/geo/ query/acc.cgi?acc=GSE13535), a public functional genomics data repository. The GSE13535 expression profile is based on the GPL1355 platform ([Rat230_2] Affymetrix Rat Genome 2302.0 Array). The specimens were acquired at 2 and $18 \mathrm{~h}$ after microsphere-injection in a rat model of PE (18), and the vehicle-treated samples were considered a control group. A total of 22 specimens were available for the present study, including three vehicle-treated specimens acquired at $2 \mathrm{~h}$, eight microsphere-injection specimens acquired at $2 \mathrm{~h}$, three vehicle-treated specimens acquired at $18 \mathrm{~h}$ and eight microsphere-injection specimens acquired at $18 \mathrm{~h}$.

Data preprocessing and screening of DEGs. The derived genetic data was initially analyzed using affy package (19) in $\mathrm{R}$ software, and the array files were converted into gene expression spectrum data. Robust multi-array average (20) was then used to standardize the expression spectrum data. Furthermore, the limma package (21) was used to calculate and analyze the DEGs of the sample groups, as compared with the control group, and the Bayes method was applied for a multiple range test. The DEGs were screened based on a cut-off value, which was set at $\mid \log \mathrm{FCl}$ (fold change) $>1$ and $\mathrm{P}<0.05$.

Kyoto Encyclopedia of Genes and Genomes (KEGG) pathways enrichment analysis. Database for Annotation Visualization and Integrated Discovery (DAVID) (22) online analytical tools were used to perform KEGG pathway analysis on the DEGs obtained from the experimental specimens. The pathways with a $\mathrm{P}<0.05$ were considered to indicate significant pathways in which the DEGs were involved.
PPI network construction. The PPI networks of the DEGs at 2 and $18 \mathrm{~h}$ after PE initiation were constructed from PPI pairs whose protein interaction scores were $>0.4$, as determined by the Search Tool for the Retrieval of Interacting Genes/Proteins (23) online tool. The protein interaction scores were calculated using the following formula (24):

$$
\begin{aligned}
S(\mathrm{e}(x, y)) & =f(\operatorname{diff}(x), \operatorname{corr}(x, y), \operatorname{diff}(y)) \\
& =-2 \sum_{i=1}^{k} \log _{e}\left(p_{i}\right)
\end{aligned}
$$

Where diff $(x)$ and diff $(y)$ are differential expression assessments of gene $\mathrm{x}$ and gene $\mathrm{y}$, respectively. Corr $(x, y)$ represents the correlation between gene $x$ and gene $y . \mathrm{k}=3, \mathrm{p}_{1}$ and $\mathrm{p}_{2}$ are the $\mathrm{P}$-values of the differential expression of the two nodes, and $\mathrm{p}_{3}$ is the $\mathrm{P}$-value of their co-expression (24). Visualization of the PPI network was acquired using the igraph package (25) in R software.

Functional module analysis. Functional modules of the networks were explored using the ClusterONE plug-in of Cytoscape software (26). The top three modules of the network at $18 \mathrm{~h}$ post-PE initiation were screened under the condition of minimum size $=6$ and minimum density $=0.05$. The DEGs in the top three modules were then analyzed by Gene Ontology (GO) functional enrichment analysis.

\section{Results}

Screening DEGs at two time points. The standardization of the expression spectrum data showed a good result (Fig. 1). A total of 80 and 346 DEGs were identified 2 and $18 \mathrm{~h}$ following injection with the microsphere, respectively, as compared with the vehicle-treated specimens. There were 47 DEGs specifically at $2 \mathrm{~h}, 313$ DEGs specifically at $18 \mathrm{~h}$, and 33 genes that were differentially expressed at 2 and $18 \mathrm{~h}$ (Fig. 2). The most significantly upregulated and downregulated genes at $2 \mathrm{~h}$ were chemokine (C-C motif) ligand (CCL) 2 and Retnla (resistin like $\alpha$ ), and at $18 \mathrm{~h}$ were tissue factor pathway inhibitor 2 (TFPI2) and cytochrome P450 family 2 subfamily E polypeptide 1 (Cypla1). 


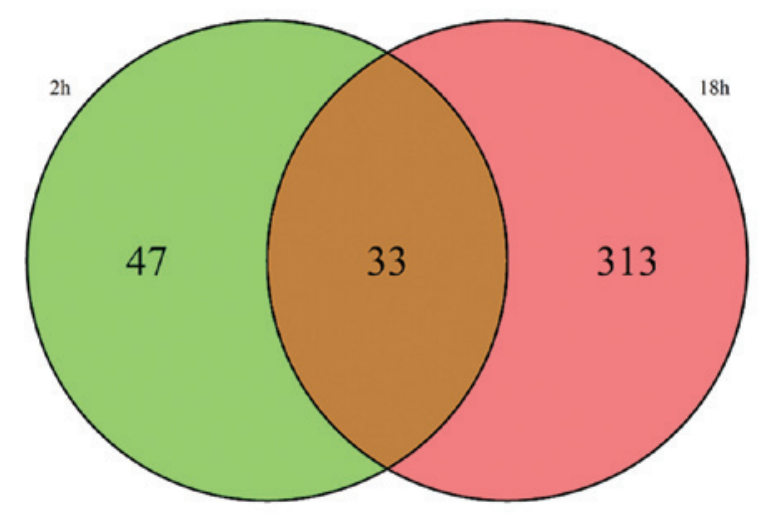

Figure 2. Venn diagram of the differentially expressed genes (DEGs) identified 2 and $18 \mathrm{~h}$ after pulmonary embolism (PE) initiation. Green represents the DEGs of PE at $2 \mathrm{~h}$, red represents the DEGs of PE at $18 \mathrm{~h}$, and brown represents the DEGs observed at both 2 and $18 \mathrm{~h}$.

A

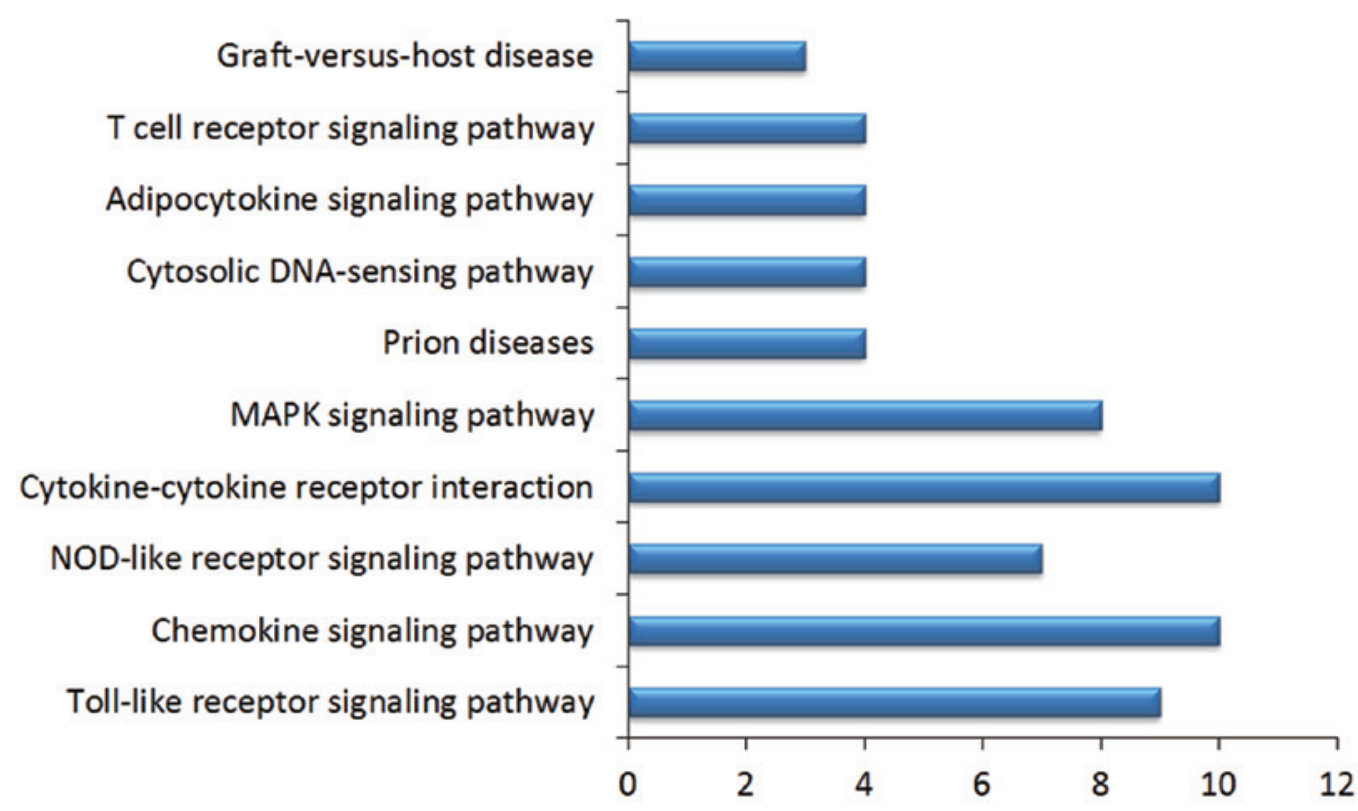

B

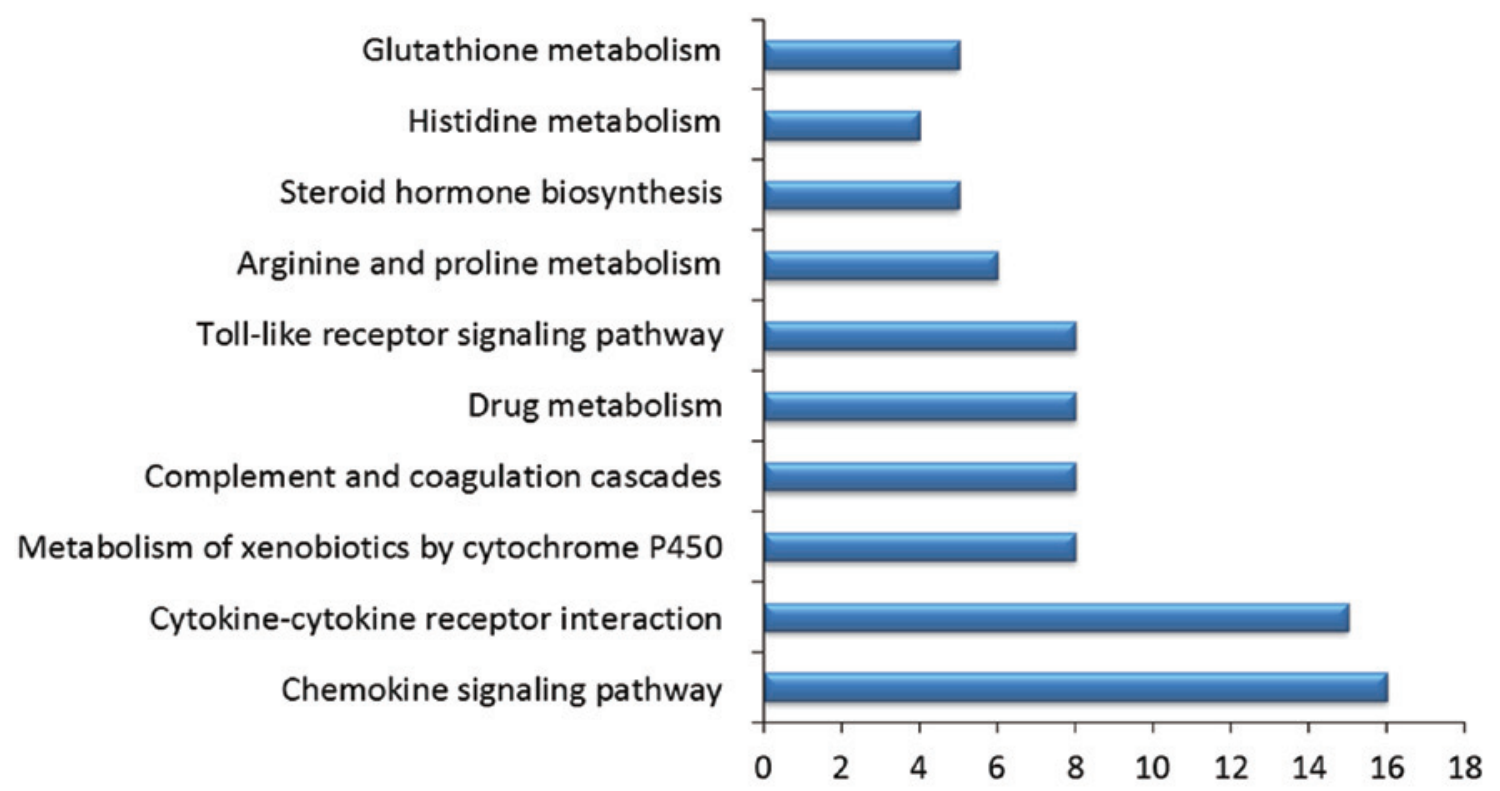

Figure 3. The enriched Kyoto Encyclopedia of Genes and Genomes (KEGG) pathways of the differentially expressed genes (DEGs) in pulmonary emblolism (PE) specimens at the different time points: (A) $2 \mathrm{~h}$ and (B) $18 \mathrm{~h}$. The X-axis represents the amounts of DEGs, and the Y-axis represents enriched KEGG pathways. The blue bars represent the P-value, which was determined using the Database for Annotation Visualization and Integrated Discovery. The P value is increased from the bottom up. 
A

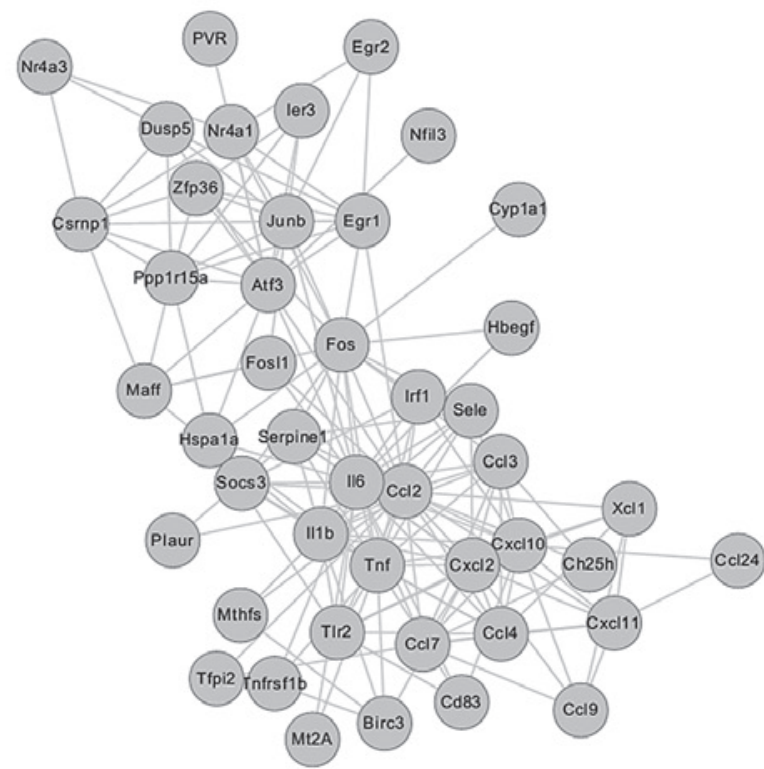

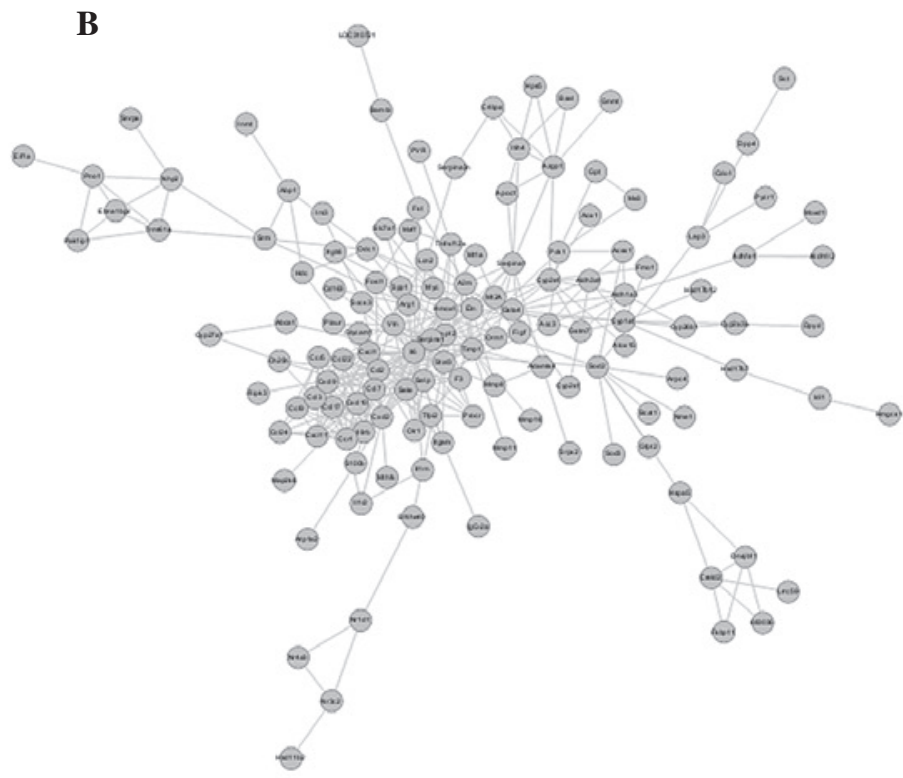

Figure 4. Protein-protein interaction networks of the differentially expressed genes (DEGs) in pulmonary embolism at the different time points (A) $2 \mathrm{~h}$ and (B) $18 \mathrm{~h}$. The nodes indicate the DEGs and the edges indicate the interactions between two genes.

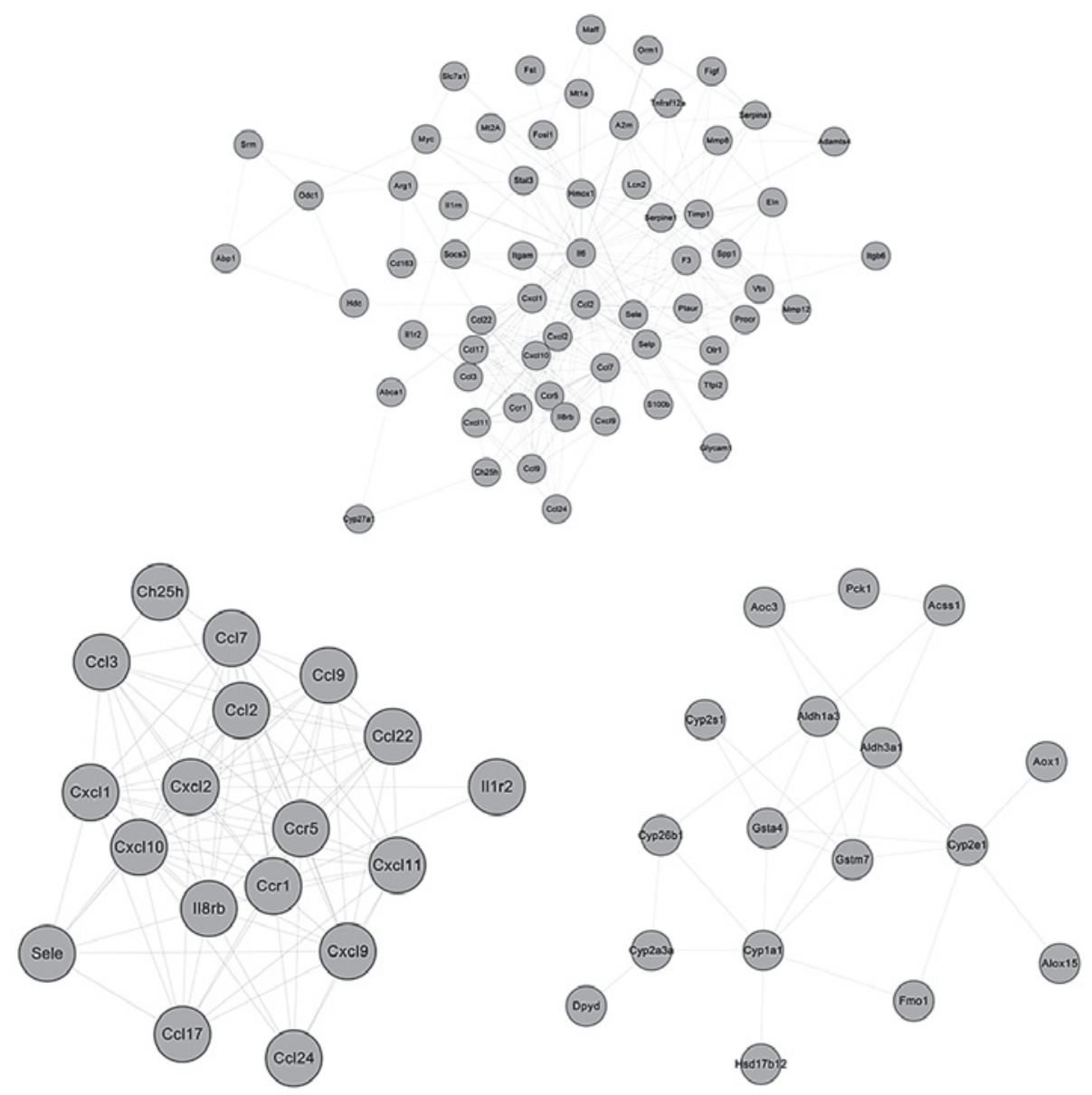

Figure 5. Top three modules in the protein-protein interaction network of the differentially expressed (DEGs) in pulmonary embolism at 18 h. The nodes indicate the DEGs and the edges indicate the interactions between two genes.

Pathway analysis of DEGs. The KEGG pathway analysis at $2 \mathrm{~h}$ after PE initiation, identified DEGs that were significantly enriched in the toll-like receptor signaling pathway, chemokine signaling pathway, cytokine-cytokine receptor interaction and Ras-mitogen-activated protein kinase (MAPK) signaling pathway (Fig. 3A). Two of these pathways, 
Table I. Biological function analysis of differentially expressed genes in the top three modules of the protein-protein interaction network.

\begin{tabular}{|c|c|c|c|c|}
\hline Module & ID & Term & Count & P-value \\
\hline \multirow[t]{10}{*}{1} & GO:0009611 & Response to wounding & 17 & $1.52 \mathrm{E}-14$ \\
\hline & GO:0050900 & Leukocyte migration & 10 & $5.06 \mathrm{E}-14$ \\
\hline & GO:0006954 & Inflammatory response & 13 & $7.05 \mathrm{E}-13$ \\
\hline & GO:0008009 & Chemokine activity & 8 & $3.10 \mathrm{E}-12$ \\
\hline & GO:0042379 & Chemokine receptor binding & 8 & $3.92 \mathrm{E}-12$ \\
\hline & GO:0005615 & Extracellular space & 16 & $1.45 \mathrm{E}-11$ \\
\hline & GO:0006952 & Defense response & 14 & $5.25 \mathrm{E}-11$ \\
\hline & GO:0006935 & Chemotaxis & 9 & $9.04 \mathrm{E}-11$ \\
\hline & GO:0042330 & Taxis & 9 & $9.04 \mathrm{E}-11$ \\
\hline & GO:0044421 & Extracellular region part & 17 & $1.57 \mathrm{E}-10$ \\
\hline \multirow[t]{10}{*}{2} & GO:0008009 & Chemokine activity & 12 & $8.82 \mathrm{E}-26$ \\
\hline & GO:0042379 & Chemokine receptor binding & 12 & $1.32 \mathrm{E}-25$ \\
\hline & GO:0005125 & Cytokine activity & 12 & $2.80 \mathrm{E}-19$ \\
\hline & GO:0006955 & Immune response & 13 & $3.53 \mathrm{E}-15$ \\
\hline & GO:0042330 & Taxis & 9 & 4.29E-14 \\
\hline & GO:0006935 & Chemotaxis & 9 & 4.29E-14 \\
\hline & GO:0050900 & Leukocyte migration & 8 & $3.91 \mathrm{E}-13$ \\
\hline & GO:0005615 & Extracellular space & 12 & $1.55 \mathrm{E}-11$ \\
\hline & GO:0007626 & Locomotory behavior & 9 & $7.66 \mathrm{E}-11$ \\
\hline & GO:0006954 & Inflammatory response & 9 & $1.08 \mathrm{E}-10$ \\
\hline \multirow[t]{10}{*}{3} & GO:0055114 & Oxidation reduction & 11 & $2.45 \mathrm{E}-10$ \\
\hline & GO:0006805 & Xenobiotic metabolic process & 5 & $1.61 \mathrm{E}-08$ \\
\hline & GO:0009410 & Response to xenobiotic stimulus & 5 & 2.65E-08 \\
\hline & GO:0005506 & Iron ion binding & 7 & $1.23 \mathrm{E}-06$ \\
\hline & GO:0009055 & Electron carrier activity & 6 & $7.78 \mathrm{E}-06$ \\
\hline & GO:0005783 & Endoplasmic reticulum & 8 & 2.09E-05 \\
\hline & GO:0048037 & Cofactor binding & 6 & 3.39E-05 \\
\hline & GO:0005789 & Endoplasmic reticulum membrane & 5 & $1.18 \mathrm{E}-04$ \\
\hline & GO:0042175 & Nuclear envelope-endoplasmic reticulum network & 5 & 1.49E-04 \\
\hline & GO:0042573 & Retinoic acid metabolic process & 3 & $1.71 \mathrm{E}-04$ \\
\hline
\end{tabular}

GO, gene ontology.

the chemokine signaling pathway and the cytokine-cytokine receptor interaction pathway, were also enriched in the $18 \mathrm{~h}$ group (Fig. 3B).

Results of the PPI network. The PPI network of DEGs $2 \mathrm{~h}$ after PE initiation consisted of 45 nodes and 170 edges, whereas 137 nodes and 378 edges were included in the PPI network of the $18 \mathrm{~h}$ group (Fig. 4). In the network of DEGs from the $2 \mathrm{~h}$ group, the top five highest degree nodes (Fig. 4A) included CCL2, interleukin (IL)-6, tumor necrosis factor (TNF), FBJ osteosarcoma oncogene (FOS) and chemokine (C-X-C motif) ligand (CXCL) 10. The top five nodes in the PPI network at 18 h (Fig. 3B) were IL-6, CCL2, heme oxygenase 1 (HMOX1), TIMP metallopeptidase inhibitor 1 (TIMP1) and serpin peptidase inhibitor clade E member 1 (SERPINE1).
Module analysis in the network. Since there were more nodes and edges in the PPI network at $18 \mathrm{~h}$, as compared with at $2 \mathrm{~h}$, ClusterONE was used to perform a module analysis for the network at $18 \mathrm{~h}$ only. The top three modules, which had the lowest P-values, are listed in Fig. 5. In module 1, the highest degree node was IL-6 (degree 40), and the second highest was CCL2 (degree 30). In module 2, there were seven nodes with the highest degree of 13, including CCL7, CXCL10, CXCL11, IL-8RB, CXCL2, CCL2, and chemokine (C-C motif) receptor 1 (CCR1).

Functional enrichment analysis of modules. Following a functional enrichment analysis of the DEGs in the top three modules, the top 10 GO terms were listed (Table I). The DEGs of module 1 were significantly enriched in response to 
wounding $(\mathrm{P}=1.52 \mathrm{E}-14)$, leukocyte migration $(\mathrm{P}=5.06 \mathrm{E}-14)$ and the inflammatory response $(\mathrm{P}=7.05 \mathrm{E}-13)$; the DEGs in module 2 were markedly enriched in chemokine activity $(\mathrm{P}=8.82 \mathrm{E}-26)$, chemokine receptor binding $(\mathrm{P}=1.32 \mathrm{E}-25)$ and the immune response $(\mathrm{P}=3.53 \mathrm{E}-15)$; and the $\mathrm{DEGs}$ in module 3 were enriched in oxidation reduction $(\mathrm{P}=2.45 \mathrm{E}-10)$, xenobiotic metabolic process $(\mathrm{P}=1.61 \mathrm{E}-08)$ and iron ion binding $(\mathrm{P}=1.23 \mathrm{E}-06)$.

\section{Discussion}

Due to the high mortality rate associated with PE, understanding the mechanisms of PE pathogenesis is required, in order to identify potential therapeutic targets. The present study used a gene expression profile, downloaded from GEO, to analyze the possible functions and pathways of DEGs in PE. The most significantly upregulated and downregulated genes at $2 \mathrm{~h}$ after PE initiation were CCL2 and Retnla, and at $18 \mathrm{~h}$ were TFPI2 and Cypla1, respectively. CCL2 was previously shown to be downregulated in a mouse model of DVT, which may be treated with IL-6 antibodies (27). Furthermore, the mRNA expression levels of chemokines, such as CCL2 and CCL7, have been shown to be significantly lower in patients with PE, as compared with controls (14). However, these findings were not in concordance with the results of the present study. CCL2 was upregulated at $2 \mathrm{~h}$ after microsphere injection, suggesting that the expression of CCL2 may be associated with the duration of PE; however, this requires further investigation. Previous studies have suggested that TFPI may contribute to the efficacy of low molecular weight heparins (28), which are an effective treatment for PE (29). The present study observed an upregulation of TFPI2 in PE, indicating that TFPI2 may be a target gene for the treatment of PE. The results of the present study identified novel perspectives that CCL2 may exhibit a role in the pathogenesis of PE, as well as Retnla, TFPI2 and Cypla1.

The chemokine signaling and cytokine-cytokine receptor interaction pathways were enriched 2 and $18 \mathrm{~h}$ after PE initiation, as determined by KEGG pathway analysis of the DEGs. Furthermore, the toll-like receptor signaling, nucleotide-binding oligomerization domain (NOD)-like receptor signaling and MAPK signaling pathways were all significantly enriched in the $2 \mathrm{~h}$ group. Cytokine-cytokine receptor interactions have previously been reported to be crucial during immunological and inflammatory responses to disease $(30,31)$. In addition, the toll-like receptor signaling pathway may activate immune response-related pathways (32), and has been shown to be associated with chronic obstructive pulmonary disease (33). Therefore, it seems rational to speculate that the toll-like receptor signaling pathway may have a role in PE. Furthermore, the NOD-like receptor signaling pathway has been demonstrated to be associated with coagulation and inflammation (34). Further studies are required to determine whether the NOD-like receptor signaling pathway has a role in the early onset of PE.

The following DEGs: CCL2, IL-6, TNF, FOS, CXCL10, HMOX1, TIMP1 and SERPINE1, were the highest degree nodes in the PPI network. CCL7, CXCL11, Il8rb, CXCL2 and CCR1 were also observed to be significantly altered in the module analysis. The expression levels of certain TNF superfamily members have previously been shown to be significantly upregulated in patients with PE (14). Chemokines can be categorized into four classes: $\mathrm{CXC}, \mathrm{CC}, \mathrm{C}$ and $\mathrm{CX} 3 \mathrm{C}$, according to their structure. Lv et al (14), demonstrated that the mRNA expression levels of the following chemokines: CCL2, CCL7, CXCL2, CXCL10 and CXCL11, were significantly upregulated in patients with PE (14), this finding is concordant with the results of the present study. These results imply that chemokines and the TNF family may have important roles in PE. In addition, the inflammatory, defense and immune responses were significantly enriched in the DEGs, as determined by GO terms analysis. It has previously been reported that patients with PE possess a lowered immune function $(35,36)$, and CD137L, a member of the TNF family, which is important in immune regulation (37), was significantly altered in the present study. Inflammation may also have a role in venous thromboembolism, which is comprised of DVT and PE, as it has been reported that patients with DVT present the four cardinal signs of inflammation $(38,39)$. In the present study, the inflammatory response pathway was enriched, further indicating that inflammation may be closely associated with PE.

In conclusion, the present study analyzed the DEGs profiles of PE using a computational bioinformatics approach. A number of key genes, including CCL2 and CXCL10, TNF, Retnla, TFPI2 and Cypla1, were identified as having potentially crucial roles in PE. Chemokine signaling, chemokine activity pathway and inflammatory response may also be associated with the development of PE. The present study provides a novel perspective regarding the mechanisms of PE. However, further verification experiments and mechanistic studies on the process of PE are required.

\section{Acknowledgements}

The authors of the present study would like to thank everyone who contributed to the research.

\section{References}

1. Garg K, Welsh CH, Feyerabend AJ, et al: Pulmonary embolism: diagnosis with spiral CT and ventilation-perfusion scanning - correlation with pulmonary angiographic results or clinical outcome. Radiology 208: 201-208, 1998.

2. Abdel-Razeq H, Qari M, Kristensen J, et al: Guidelines for diagnosis and treatment of deep venous thrombosis and pulmonary embolism. Methods Mol Med 93: 267-292, 2004.

3. Baue AE: Prevention of venous thrombosis and pulmonary embolism. JAMA 256: 757, 1986.

4. Carson JL, Kelley MA, Duff A, et al: The clinical course of pulmonary embolism. N Engl J Med 326: 1240-1245, 1992.

5. Weg JG: Pulmonary embolism: diagnosis and treatment. Appl Cardiopulm Pathophysiol 2: 23-25, 1988.

6. Dobkin J and Reichel J: Pulmonary embolism: diagnosis and treatment. Cardiol Clin 5: 577-582, 1987.

7. Prieto AR and Penner J: Pulmonary Embolism Diagnosis and Treatment. In: Peripheral Vascular Disease: Basic Diagnostic and Therapeutic Approaches. Abela GS (ed). 1st edition. Lippincott Williams \& Wilkins, Philadelphia, PA, p461, 2004.

8. Stein PD, Athanasoulis C, Alavi A, et al: Complications and validity of pulmonary angiography in acute pulmonary embolism. Circulation 85: 462-468, 1992.

9. Anderson FA Jr, Wheeler HB, Goldberg RJ, et al: A population-based perspective of the hospital incidence and case-fatality rates of deep vein thrombosis and pulmonary embolism. The Worcester DVT Study. Arch Intern Med 151: 933-938, 1991.

10. Sutton GC, Hall RJ and Kerr IH: Clinical course and late prognosis of treated subacute massive, acute minor, and chronic pulmonary thromboembolism. Br Heart J 39: 1135-1142, 1977. 
11. Koster T, Rosendaal FR, de Ronde H, et al: Venous thrombosis due to poor anticoagulant response to activated protein C: Leiden Thrombophilia Study. Lancet 342: 1503-1506, 1993.

12. Nizankowska-Mogilnicka E, Adamek L, Grzanka P, et al: Genetic polymorphisms associated with acute pulmonary embolism and deep venous thrombosis. Eur Respir J 21: 25-30, 2003.

13. Bounameaux H: Factor V Leiden paradox: risk of deep-vein thrombosis but not of pulmonary embolism. Lancet 356: 182-183, 2000.

14. Lv W, Duan Q, Wang L, et al: Gene expression levels of cytokines in peripheral blood mononuclear cells from patients with pulmonary embolism. Mol Med Rep 7: 1245-1250, 2013.

15. Szczeklik A, Sanak M, Jankowski M, et al: Mutation A1298C of methylenetetrahydrofolate reductase: risk for early coronary disease not associated with hyperhomocysteinemia. Am J Med Genet 101: 36-39, 2001.

16. Zoller B, García de Frutos P and Dahlbäck B: A common $4 \mathrm{G}$ allele in the promoter of the plasminogen activator inhibitor-1 (PAI-1) gene as a risk factor for pulmonary embolism and arterial thrombosis in hereditary protein $\mathrm{S}$ deficiency. Thromb Haemost 79: 802-807, 1998.

17. Gonzalez-Juarrero M, Hattle JM, Izzo A, et al: Disruption of granulocyte macrophage-colony stimulating factor production in the lungs severely affects the ability of mice to control Mycobacterium tuberculosis infection. J Leukoc Biol 77: 914-922, 2005.

18. Barrett T, Wilhite SE, Ledoux P, et al: NCBI GEO: archive for functional genomics data sets - update. Nucleic Acids Res 41: D991-D995, 2013

19. Gautier L, Cope L, Bolstad BM and Irizarry RA: affy - analysis of Affymetrix GeneChip data at the probe level. Bioinformatics 20 : 307-315, 2004

20. Qu Y, He F and Chen Y: Different effects of the probe summarization algorithms PLIER and RMA on high-level analysis of Affymetrix exon arrays. BMC Bioinformatics 11: 211, 2010.

21. Diboun I, Wernisch L, Orengo CA and Koltzenburg M: Microarray analysis after RNA amplification can detect pronounced differences in gene expression using limma. BMC Genomics 7: 252, 2006.

22. Huang da W, Sherman BT, Tan Q, et al: The DAVID Gene Functional Classification Tool: a novel biological module-centric algorithm to functionally analyze large gene lists. Genome Biol 8: R183, 2007.

23. Franceschini A, Szklarczyk D, Frankild S, et al: STRING v9.1: protein-protein interaction networks, with increased coverage and integration. Nucleic Acids Res 41: D808-D815, 2013.
24. Liu ZP, Wang Y, Zhang XS, Chen L: Identifying dysfunctional crosstalk of pathways in various regions of Alzheimer's disease brains. BMC Syst Biol 4: S11, 2010.

25. Mora A and Donaldson IM: iRefR: an R package to manipulate the iRefIndex consolidated protein interaction database. BMC Bioinformatics 12: 455, 2011.

26. Zaki N, Berengueres J and Efimov D: Detection of protein complexes using a protein ranking algorithm. Proteins 80: 2459-2468, 2012

27. Rodriguez AL, Wojcik BM, Wrobleski SK, et al: Statins, inflammation and deep vein thrombosis: a systematic review. J Thromb Thrombolysis 33: 371-382, 2012.

28. Mousa SA, Bozarth J and Barrett JS: Pharmacodynamic properties of the low molecular weight heparin, tinzaparin: effect of molecular weight distribution on plasma tissue factor pathway inhibitor in healthy human subjects. J Clin Pharmacol 43: 727-734, 2003.

29. Simonneau G, Sors H, Charbonnier B, et al: A comparison of low-molecular-weight heparin with unfractionated heparin for acute pulmonary embolism. The THESEE Study Group. Tinzaparine ou Heparine Standard: Evaluations dans l'Embolie Pulmonaire. N Engl J Med 337: 663-669, 1997.

30. Turrin NP and Plata-Salamán CR: Cytokine-cytokine interactions and the brain. Brain Res Bull 51: 3-9, 2000.

31. Grotzinger J: Molecular mechanisms of cytokine receptor activation. Biochim Biophys Acta 1592: 215-223, 2002.

32. Barton GM and Medzhitov R: Toll-like receptor signaling pathways. Science 300: 1524-1525, 2003.

33. Sethi $\mathrm{S}$ and Murphy TF: Infection in the pathogenesis and course of chronic obstructive pulmonary disease. N Engl J Med 359: 2355-2365, 2008

34. Lipinski S, Bremer L, Lammers T, et al: Coagulation and inflammation. Molecular insights and diagnostic implications. Hamostaseologie 31: 94-104, 2011.

35. Wang L, Song H, Gong Z, Duan Q and Liang A: Acute pulmonary embolism and dysfunction of CD3+ CD8+ T cell immunity. Am J Respir Crit Care Med 184: 1315, 2011.

36. Haoming S, Lemin $\mathrm{W}$, Zhu G, et al: T cell-mediated immune deficiency or compromise in patients with CTEPH. Am J Respir Crit Care Med 183: 417-418, 2011.

37. Vinay DS and Kwon BS: Immunotherapy targeting 4-1BB and its ligand. Int J Hematol 83: 23-28, 2006.

38. Fox EA and Kahn SR: The relationship between inflammation and venous thrombosis. Thromb Haemost 94: 362-365, 2005.

39. van Aken BE, den Heijer M, Bos GM, van Deventer SJ and Reitsma PH: Recurrent venous thrombosis and markers of inflammation. Thromb Haemost 83: 536-539, 2000. 